\title{
Anosmia and dysgeusia among COVID-19 patients are associated with low levels of serum Glucagon-like peptide 1 (GLP-1)
}

\author{
Eli Ben-Chetrit ${ }^{1}$, Ami Ben Yaa'cov ${ }^{1}$, Ahamad Quiteineh ${ }^{1}$, Ohad Atia ${ }^{1}$, Eran Regev ${ }^{1}$, Eyal \\ Shteyer $^{1}$, and Rizan Nashef ${ }^{1}$ \\ ${ }^{1}$ Shaare Zedek Medical Center
}

July 9, 2021

\begin{abstract}
Purpose: Anosmia and dysgeusia (AD) are common among COVID-19 patients. These symptoms are not frequently associated with rhinorrhea or nasal congestion and the underlying mechanism is unclear. Previous reports suggested that Glucagon-like peptide-1 (GLP-1) signaling plays a role in the modulation of olfaction and geusia. We aimed to assess the correlation between GLP-1 and COVID-19-associated AD. Methods: Blood samples obtained from COVID-19 patients with and without AD were tested for serum GLP-1 levels using enzyme-linked immunosorbent assay (ELISA). A second control group comprised of COVID19-negative volunteers. Results: Forty-nine subjects were included in the study. Nineteen were positive for COVID-19. Of the 19 patients, ten had AD and nine declined such complaints. Age and basic metabolic rate were similar among all study groups. Serum GLP-1 levels were significantly lower among patients with AD as compared with patients without AD and COVID19-negative individuals $(1820 \mathrm{pg} / \mathrm{ml}$ vs $3536 \mathrm{pg} / \mathrm{ml}$ vs $3014 \mathrm{pg} / \mathrm{ml}$, respectively, $\mathrm{p}<0.02)$. Conclusion: COVID-19 patients who reported of $\mathrm{AD}$ had lower serum levels of GLP-1 as compared with those lacking AD symptoms and COVID-19-negative individuals. These results suggest that GLP-1 may be involved in the pathogenesis of AD. However, further larger scale studies should corroborate our findings
\end{abstract}

Anosmia and dysgeusia among COVID-19 patients are associated with low levels of serum Glucagon-like peptide 1 (GLP-1)

Abstract

Purpose: Anosmia and dysgeusia (AD) are common among COVID-19 patients. These symptoms are not frequently associated with rhinorrhea or nasal congestion and the underlying mechanism is unclear. Previous reports suggested that Glucagon-like peptide-1 (GLP-1) signaling plays a role in the modulation of olfaction and geusia. We aimed to assess the correlation between GLP-1 and COVID-19-associated AD.

Methods: Blood samples obtained from COVID-19 patients with and without AD were tested for serum GLP-1 levels using enzyme-linked immunosorbent assay (ELISA). A second control group comprised of COVID-19-negative volunteers.

Results: Forty-nine subjects were included in the study. Nineteen were positive for COVID-19. Of the 19 patients, ten had AD and nine declined such complaints. Age and basic metabolic rate were similar among all study groups. Serum GLP-1 levels were significantly lower among patients with AD as compared with patients without AD and COVID-19-negative individuals $(1820 \mathrm{pg} / \mathrm{ml}$ vs $3536 \mathrm{pg} / \mathrm{ml}$ vs $3014 \mathrm{pg} / \mathrm{ml}$, respectively, $\mathrm{p}<0.02)$.

Conclusion: COVID-19 patients who reported of AD had lower serum levels of GLP-1 as compared with those lacking AD symptoms and COVID-19-negative individuals. These results suggest that GLP-1 may be involved in the pathogenesis of AD. However, further larger scale studies should corroborate our findings 
Key words: Anosmia; dysgeusia, COVID-19; Glucagon-like peptide-1

Introduction

Olfactory and taste disorders are known to be related to a wide range of viral infections including SARS$\mathrm{CoV}-2$. However, the pathophysiological mechanism has yet to be determined. Many viruses lead to anosmia and/or dysgeusia (AD) through an inflammatory reaction of the nasal mucosa leading to rhinorrhea and nasal congestion. However, among COVID-19 patients, especially of mild severity, AD may present as an isolated complaint (1). In a recent study, of 2013 COVID-19 patients, 1754 patients (87\%) reported loss of smell, whereas 1136 (56\%) reported taste dysfunction. Both complaints were more common among mild COVID-19 patients (2). Angiotensin-converting enzyme-2 (ACE2) is the main host cell receptor that mediates SARS$\mathrm{CoV}-2$ invasion to cells, and is widely expressed in multiple tissues including the epithelial cells of the oral mucosa. In fact, this receptor was highly enriched in epithelial cells of tongue (3). An inflammatory response mediated by cytokines leading to oral and olfactory mucosal injury was suggested as a possible mechanism for $\mathrm{AD}(4)$.

Interestingly, the signaling pathway of taste modulation and olfaction involves several ligands, among them is glucagon-like peptide-1 (GLP-1) (5). GLP-1 is a pleiotropic gut hormone with numerous metabolic functions and has a main role in glucose homeostasis. GLP-1 is released in the gut in response to nutrient ingestion and its activity is regulated by the enzyme dipetidyl-peptidase- 4 . The taste cells, particularly L-cells, produce GLP-1 among other hormones (6). Several reports suggest that GLP-1 functions as a neurotransmitter mediating taste and olfaction $(5,7,8)$. Mice lacking the GLP-1 receptor have significantly reduced taste sensitivity to sweeteners (7). Pre-pro-glucagon (the precursor of GLP-1) and GLP-1 receptor mRNAs have been identified in cell layers in the olfactory bulb suggesting that GLP-1 may mediate information flow from the olfactory epithelium to the brain (8).

In view of its role in modulation of gustatory and olfaction functions, we aimed to examine the association between serum GLP-1 levels among COVID-19 patients reporting AD versus patients and COVID-19-negative individuals with intact olfaction and taste.

Materials and Methods

The study took place in the COVID-19 wards of Shaare Zedek Medical center (SZMC), Jerusalem, Israel. We established a new bio-bank of various blood-derived products from hospitalized COVID-19 patients. COVID-19 was confirmed based on PCR positive nasopharyngeal swab specimen tested for SARS-CoV-2. Our study included COVID-19 confirmed patients with AD symptoms (group A), without AD symptoms (group B) as well as COVID-19-negative asymptomatic subjects that served as a second control group (group C). Clinical and demographic data was retrieved from the computerized medical records or by interviewing the study participants. Those included: age, sex, comorbidities, presence of anosmia/dysosmia and or/ dysgeusia and patients' medications. Severity of COVID-19 was assessed using the NIH guidelines (https://www.covid19treatmentguidelines.nih.gov).

Blood samples were collected from COVID-19-confirmed patients (based on nasopharyngeal PCR testing) and from COVID-19-negative individuals in $5 \mathrm{~mL}$ CAT tubes (456018, Vacuette; Greiner Bio-one). Samples were centrifuged at $1800 \mathrm{x} \mathrm{g}, \mathrm{R} / \mathrm{T}$ for 20 minutes according to protocol, sera were separated and aliquots were stored in a dedicated $-80^{\circ} \mathrm{c}$ COVID-19 freezer. Serum GLP-1 levels were measured using a commercial enzyme-linked immunosorbent assay (ELISA) according to the manufacturer instructions (abcam, Cambridge, UK). Differences between groups were assessed using Chi-square test for categorical variables and Welch analysis of variance (ANOVA) test for continuous variables with skewed distribution. $\mathrm{P}$ value $<0.05$ was considered statistically significant. Informed consent was obtained from all participants. The study was approved by the Institutional Review Board of SZMC.

Results

Forty-nine subjects were included in the study. Nineteen were COVID-19-confirmed hospitalized patients. Of them, $11(57.9 \%)$ had mild disease, $3(15.8 \%)$ had moderate disease and 5 (26.3\%) had severe illness. 
One patient with severe COVID-19 died. Overall, 9 COVID-19 patients had complained of AD (group A) and 10 had no AD symptoms (group B). A third group of 30 asymptomatic COVID-19 negative subjects (group C) served as a second control group. The demographic and clinical characteristics of all subjects are shown in Table. None of the patients with AD had rhinitis or rhinorrhea.

Age and BMI did not significantly differ between groups. The median age of patients in groups A, B and $\mathrm{C}$ was 52 (range 34-62), 64 (range 40-80) and 60 years (range 40-69), respectively ( $\mathrm{p}=0.06$ ); with male predominance $(57.1 \%)$ in all groups. Height and weight data was missing in 5 subjects. COVID-19 patients were more obese than COVID-19 negative controls (Table).

Type 2 diabetes mellitus was reported in 22.2\% (2/9) of patients in group A, 40\% (4/10) in group B and $3.3 \%(1 / 30)$ in group C (Table). One diabetic patient with intact olfaction and geusia was treated with GLP-1 receptor analogue (liraglutide). Prevalence of co-morbidities aside of diabetes (including primarily hypertension, hypothyroidism, and hyperlipidemia) was similar among Groups A and B (Table).

The mean serum GLP-1 levels among group A patients were significantly lower as compared with GLP-1 levels in groups B and C $(1820 \pm 226 \mathrm{pg} / \mathrm{ml}$ vs. $3536 \pm 449 \mathrm{pg} / \mathrm{ml}$ and $3014 \pm 161 \mathrm{pg} / \mathrm{ml}, \mathrm{p}<0.002$, respectively) (Figure 1). Serum GLP-1 levels among groups B and C were similar ( $\mathrm{p}=0.65)$. GLP-1 levels were also found significantly low among Group A patients vs Groups B and C after adjustment to age, sex, diabetes, and BMI $(p=0.02)$. No correlation was found between severity of disease and serum GLP-1 levels.

\section{Discussion}

Among all symptoms arise from COVID-19 infection the changes in taste and smell, although not lifethreatening, seem the most bothersome to many patients. However, the pathophysiology of anosmia and dysgeusia is not fully elucidated. In this study we examined the association of serum GLP-1 levels and AD among COVID-19 patients. GLP-1 levels were significantly lower among COVID-19 patients with AD as compared with patients without AD and COVID-19-negative subjects.

Prevalence of smell and/or taste impairment is high among COVID-19 patients (2, 9-11). A proposed mechanism for this chemo-sensitive dysfunction was local inflammation and cytokine release in the oral and olfactory mucosa induced by SARS-CoV-2 tissue invasion (12). The high expression of ACE2 in the oral mucosa, especially the tongue and taste buds, as well as the nasal cavity olfactory epithelium supports this notion $(3,13)$. However, this theory is questionable in mild COVID-19 cases, where the inflammatory response is less robust and rhinorrhea or nasal congestion are absent (14). Indeed, these symptoms were not commonly reported in our cohort.

Others suggested that the interaction of SARS-CoV-2 with sialic acid receptors on taste buds, impairs vital components of the salivary mucin. Reduced sialic acid in the saliva may accelerate degradation of the gustatory particles $(12,15)$. However, this does not explain dysosmia.

Our findings may point to another intriguing mechanism of AD, which may be related to the gut hormone GLP-1 (5). Aside of its major role as an incretin, enhancing insulin secretion and maintaining energy homeostasis, GLP-1 has been reported to carry out protective and regulatory effects in numerous tissues, including heart, adipose, muscles, bones, kidneys, liver, lungs, and brain as well as the olfactory epithelium and the taste buds on the tongue $(16,17)$. In fact, it functions as a neurotransmitter, coordinating communication between the peripheral organs and the brain $(5,18,19)$. GLP-1 modulates taste sensitivity and affects taste preferences $(5,18,19)$. Expression of GLP-1 was reported in two populations of taste cells (Type II and III cells), and its receptor has been identified in taste nerve fibers in mice and rodents (18). GLP-1 producing cells and GLP-1 receptors have also been found in the olfactory bulb (20).

In view of the above literature and in-line with our findings, we propose that low serum levels of GLP-1 may contribute to anosmia/hyposmia and dysgeusia among COVD-19 patients.

No previous reports have examined the association between GLP-1 and olfaction and geusia during viral infections, and particularly among COVID-19 patients. 
The decrease in GLP-1 may be explained either by increased degradation or decreased production of the hormone due to local inflammation in the oral and nasal cavities. Further research at the tissue and cellular level may shed light on this notion.

Our study has limitations. First, the retrospective design has inherent limitations including recall bias. Second, no histopathological analyses of samples from the oral and nasal cavity were performed. Third, due to the small sample size, our findings may not be generalizable, and subgroup analyses (based on BMI, diabetes, age) was not attainable. This may had affected our findings. However, it is unclear whether GLP-1 secretion (either basal or postprandial) is decreased or enhanced under an obese, or diabetic status (21). Importantly, BMI was similar among the study groups and only one patient in the COVID-19-negative group was treated with GLP-1 analogue.

It would be of interest to examine the degree of AD among diabetic COVID-19 patients who are treated with GLP-1 analogues or among patients post-bariatric surgery. Both groups have relatively high GLP-1 levels.

In summary, our preliminary findings point to a possible mechanism by which decreased GLP-1 may be involved in impairment of olfaction and geusia among COVID-19 patients. Further research is required to conclude a causal association.

Declarations:

Funding - the study was funded by Shaare Zedek Scientific Ltd. (Mada'it)

Conflicts of interest - none to declare

Availability of data and material - raw data will be uploaded to Research Square

Code availability - not applicable

Ethics approval - the study was approved by the SZMC institutional board, approval number SZMC-02-0278

Consent to participate - all study patients signed an informed consent permitting collection of blood samples for research purposes.

Consent for publication - all authors permit publication of the study by the publisher

References

1. Lechien JR, Chiesa-Estomba CM, De Siati DR, Horoi M, Le Bon SD, Rodriguez A et al. Olfactory and gustatory dysfunctions as a clinical presentation of mild-to-moderate forms of the coronavirus disease (COVID-19): a multicenter European study. Eur Arch Otorhinolaryngol. 2020 ;277:2251-2261.

2. Lechien JR, Chiesa-Estomba CM, Hans S, Barillari MR, Jouffe L, Saussez S. Loss of Smell and Taste in 2013 European Patients With Mild to Moderate COVID-19. Ann Intern Med. 2020 ;173:672-675.

3. Xu H, Zhong L, Deng J, Peng J, Dan H, Zeng X, Li T, Chen Q. High expression of ACE2 receptor of $2019-\mathrm{nCoV}$ on the epithelial cells of oral mucosa. Int J Oral Sci. $2020 ; 12: 8$.

4. Lozada-Nur F, Chainani-Wu N, Fortuna G, Sroussi H. Dysgeusia in COVID-19: Possible Mechanisms and Implications. Oral Surg Oral Med Oral Pathol Oral Radiol. 2020 ;130:344-346.

5. Martin B, Maudsley S, White CM, Egan JM. Hormones in the naso-oropharynx: endocrine modulation of taste and smell. Trends Endocrinol Metab. 2009 ;20:163-70.

6. Müller TD, Finan B, Bloom SR, D’Alessio D, Drucker DJ, Flatt PR et al. Glucagon-like peptide 1 (GLP-1). Mol Metab. 2019 ;30:72-130.

7. Martin B, Dotson CD, Shin YK, Ji S, Drucker DJ, Maudsley S, Munger SD. Modulation of taste sensitivity by GLP-1 signaling in taste buds. Ann N Y Acad Sci. 2009 ;1170:98-101. 
8. Merchenthaler I, Lane M, Shughrue P. Distribution of pre-pro-glucagon and glucagon-like peptide-1 receptor messenger RNAs in the rat central nervous system. J Comp Neurol. 1999 ;403:261-80.

9. Aziz M, Perisetti A, Lee-Smith WM, Gajendran M, Bansal P, Goyal H. Taste Changes (Dysgeusia) in COVID-19: A Systematic Review and Meta-analysis. Gastroenterology. 2020;159:1132-1133.

10. Spinato G, Fabbris C, Polesel J, Cazzador D, Borsetto D, Hopkins C, Boscolo-Rizzo P. Alterations in Smell or Taste in Mildly Symptomatic Outpatients With SARS-CoV-2 Infection. JAMA. 2020 ;323:20892090 .

11. Speth MM, Singer-Cornelius T, Oberle M, Gengler I, Brockmeier SJ, Sedaghat AR. Olfactory Dysfunction and Sinonasal Symptomatology in COVID-19: Prevalence, Severity, Timing, and Associated Characteristics. Otolaryngol Head Neck Surg. 2020;163:114-120.

12. Vaira LA, Salzano G, Fois AG, Piombino P, De Riu G. Potential pathogenesis of ageusia and anosmia in COVID-19 patients. Int Forum Allergy Rhinol. 2020 ;10:1103-1104.

13. Butowt R, Bilinska K. SARS-CoV-2: Olfaction, Brain Infection, and the Urgent Need for Clinical Samples Allowing Earlier Virus Detection. ACS Chem Neurosci . 2020;11:1200-1203.

14. Dev N, Sankar J, Gupta N, Meena RC, Singh C, Gupta DK, Sen MK. COVID-19 with and without anosmia or dysgeusia: A case-control study. J Med Virol. 2021;93:2499-2504.

15. Mirabelli M, Chiefari E, Puccio L, Foti DP, Brunetti A. Potential Benefits and Harms of Novel Antidiabetic Drugs During COVID-19 Crisis. Int J Environ Res Public Health. 2020;17:3664.

16. Muscogiuri G, Cignarelli A, Giorgino F, Prodam F, Santi D, Tirabassi G, Balercia G, Modica R, Faggiano A, Colao A. GLP-1: benefits beyond pancreas. J Endocrinol Invest. 2014;37: 1143-53.

17. Thiebaud N, Llewellyn-Smith IJ, Gribble F, Reimann F, Trapp S, Fadool DA. The incretin hormone glucagon-like peptide 1 increases mitral cell excitability by decreasing conductance of a voltage-dependent potassium channel. J Physiol. 2016;594: 2607-28.

18. Takai S, Yasumatsu K, Inoue M, Iwata S, Yoshida R, Shigemura N et al. Glucagon-like peptide-1 is specifically involved in sweet taste transmission. FASEB J. $2015 ; 29: 2268-80$.

19. Bagnoli E, FitzGerald U. Mitral cells and the glucagon-like peptide 1 receptor:

The sweet smell of success? Eur J Neurosci. 2019;49:422-439.

20. Fadool DA, Tucker K, Pedarzani P. Mitral cells of the olfactory bulb perform metabolic sensing and are disrupted by obesity at the level of the Kv1.3 ion channel. PLoS One. 2011;6:e24921.

21. Hira T, Pinyo J, Hara H. What is GLP-1 Really Doing in Obesity? Trends Endocrinol Metab. 2020;31:7180

\section{Hosted file}

Table.docx available at https://authorea.com/users/424656/articles/529690-anosmia-anddysgeusia-among-covid-19-patients-are-associated-with-low-levels-of-serum-glucagon-likepeptide-1-glp-1 


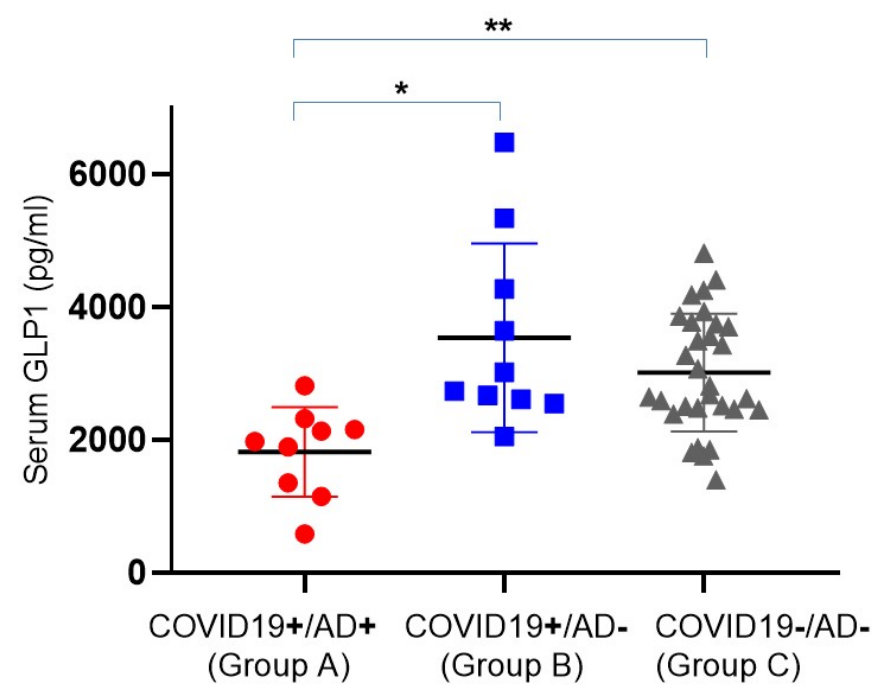

